\title{
Article
}

\section{How Effective Are Simulated Molecular-level Experiments for Teaching Diffusion and Osmosis?}

\author{
Eli Meir, ${ }^{* \dagger}$ Judith Perry, ${ }^{*}$ Derek Stal ${ }^{\dagger}$ Susan Maruca, ${ }^{\dagger}$ and Eric Klopfer*
}

\author{
*Teacher Education Program, Room 10-337, Massachusetts Institute of Technology, Cambridge, MA 02139; \\ †SimBiotic Software, 148 Grandview Court, Ithaca, NY 14850
}

Received September 15, 2004; Revised January 25, 2005; Accepted February 11, 2005

Monitoring Editor: Christopher Watters

\begin{abstract}
Diffusion and osmosis are central concepts in biology, both at the cellular and organ levels. They are presented several times throughout most introductory biology textbooks (e.g., Freeman, 2002), yet both processes are often difficult for students to understand (Odom, 1995; Zuckerman, 1994; Sanger et al., 2001; and results herein). Students have deep-rooted misconceptions about how diffusion and osmosis work, especially at the molecular level. We hypothesized that this might be in part due to the inability to see and explore these processes at the molecular level. In order to investigate this, we developed new software, OsmoBeaker, which allows students to perform inquiry-based experiments at the molecular level. Here we show that these simulated laboratories do indeed teach diffusion and osmosis and help overcome some, but not all, student misconceptions.
\end{abstract}

Keywords: undergraduate, diffusion, osmosis, simulation, misconception, biology teaching, physiology teaching

\section{INTRODUCTION}

Diffusion and osmosis are central concepts in biology, both at the cellular and organ levels. Diffusion is involved in virtually all chemical processes in living organisms, while osmosis (the diffusion of water through a semipermeable membrane) plays important roles in functions as diverse as salt balance in fish, kidney function, and the concentration of solutes in intravenous fluids. Because of their importance, both processes are mentioned several times throughout most introductory biology textbooks. For instance, Freeman (2002) discusses osmosis in chapters specifically on those topics, but also in chapters on animal form and function, gas exchange and circulation, water and electrolyte balance, and water and sugar transport in plants. Many biology classes devote a laboratory to observing osmosis as

DOI: $10.1187 /$ cbe.04-09-0049

Address correspondence to: Eli Meir (meir@simbio.com).

Eli Meir, Derek Stal, and Susan Maruca are all employees of SimBiotic Software, which is commercially distributing the product discussed in this paper. Eli Meir was involved in the assessment of the product, along with Judith Perry and Eric Klopfer. Ms. Perry and Mr. Klopfer were not associated with SimBiotic Software at the time of this work, although Ms. Perry has subsequently been hired by SimBiotic Software. well, often conducting an experiment in which the same item (e.g., onion cells, an egg, a vegetable) is placed into distilled water and into saltwater, and students observe that the item swells in the former and shrinks in the latter. These topics also introduce some mathematical thinking into introductory biology, which is becoming increasingly important (Bialek and Botstein, 2004).

Yet despite the emphasis placed on these topics, students retain much confusion and many misconceptions. Several researchers have confirmed these difficulties. Odom (1995) used a two-tier, multiple-choice test to show that many students from secondary school through college biology majors retain misconceptions in six areas, even after instruction on these topics: the particulate and random nature of matter, concentration and tonicity, the influences of life forces on diffusion and osmosis, the kinetic energy of matter, the process of diffusion, and the process of osmosis. In a previous study, Zuckerman (1994) found several common misconceptions about osmosis among outstanding high school students, including teleological explanations of osmosis, confusion between amounts and concentrations, and a misconception about molecules ceasing to move at equilibrium. As described below, we have found similar results, and noted that many of these misconceptions involve understanding how the processes of diffusion and osmosis 
work at the molecular level. For instance, whether molecules want to move in a certain direction or move randomly is not directly observable in typical laboratory experiments (nor indeed in any conceivable laboratory experiment observing actual molecules). Thus we hypothesized that these common misconceptions are due at least in part to students' inability to directly experience and observe these processes.

As part of a larger study on using simulations as tools for assessing students' understanding of biology, we designed a new program for teaching diffusion and osmosis through simulated experiments that are observable at the molecular level. A number of computer animations (e.g., Sanger et al., 2001) of both processes are available, along with a small number of simulations. For instance, the Concord Consortium has a section on diffusion and osmosis in their Molecular Workbench framework (Berenfeld et al., 2004) designed for secondary school students. The student is guided through an inquiry of diffusion and osmosis, with simulations of molecules bouncing around cell membranes embedded within a text description of the processes. Students can perform simple experiments such as adding molecules to one side but not the other, or changing the initial concentration of salt and water, and watch what happens to concentrations within and outside the simulated cell.

Simulated experiments are known to be an effective teaching tool in other areas of science (Gibbons et al., 2004; Roschelle et al., 2000), but we are not aware of any studies that specifically test whether simulations at the molecular level can aid students in overcoming their misconceptions around diffusion, osmosis, or similar topics with unobservable molecular phenomena. To explore this, we designed software that allows students to learn about both processes through conducting their own experiments and interpreting the results. In this paper we describe the misconceptions we found among students regarding diffusion and osmosis, the software (called OsmoBeaker) that we wrote as a platform for the study, and the learning gains that students achieved using this software. ${ }^{1}$ We expect that our results here may also apply to similar simulations written by other authors.

\section{METHODS}

\section{Misconceptions}

We began the study by investigating student misconceptions regarding both osmosis and diffusion. To do this, we recruited college students who had previously completed a college-level introductory biology class. Students first read several pages of introductory material on diffusion and osmosis (see below), then completed a written test consisting of questions on the subjects of osmosis and diffusion. These questions were initially validated by asking students to orally clarify their written answers during short interviews. Questions that were misinterpreted were rewritten. All questions were graded by one of the researchers. To check reliability, 20 percent of the questions were scored by another researcher and agreement was found to be $>95$ percent. All pretests and posttests were compared using two-tailed paired $t$-tests. We used several classes of written questions, including some taken from previous studies (questions 5-8 on diffusion survey from Odom, 1995; one question from Zuckerman, 1994), justified true/false, justified multiple choice, essay questions, questions for which students needed to perform calculations, and "picture questions" for which students

${ }^{1}$ Free demonstration copies of OsmoBeaker with the two labs discussed here are available from SimBiotic Software at www. simbio.com. had to either interpret a drawing or draw something themselves. Questions for which students were asked to draw pictures or perform calculations were different from the examples used in the lab (e.g., changing the configuration of membranes and cells) so students could not directly copy their work on lab questions in answering the test questions. Assessment questions are included in the Appendix. For essay and drawing questions we developed rubrics to score the answers as either no comprehension shown, partial comprehension (where appropriate), or full comprehension (scoring rubrics available upon request). For justified true/false and multiple-choice questions, we scored the student as answering correctly if both the answer and the justification were correct. We calculated overall scores for each test by assigning one point for each correct answer to questions without rubrics, and one or two points for partially or fully correct answers, respectively, to questions with rubrics (essay and drawing questions). Here we present data from the 46 pretests we collected from students before they performed one of the OsmoBeaker labs.

\section{Assessment}

As part of the study, we wrote two laboratories within OsmoBeaker, one on diffusion and the other on osmosis. Each laboratory consisted of a set of models in which students performed experiments, and a workbook that guided the students through the experiments (see below). To assess the efficacy of each laboratory, we solicited student volunteers from 11 Boston-area colleges who had taken at least one college-level introductory biology course that covered the subjects of diffusion and osmosis. The colleges ranged from large, well-known universities such as Boston University and Boston College, to smaller community colleges. Among participating students, 83 percent were freshmen or sophomores, and 71 percent were women. Of these students, 84 percent had received instruction on osmosis in their introductory college biology class, while most of the others learned about osmosis in their high school biology course. At least 50 percent had completed a wet lab on osmosis in either high school or college. While we did not formally track language background, several students spoke English as a second language, with all possessing good command of English (a requirement for participation). Participants met with a researcher for a 2 -h session and were financially compensated for their time.

In performing our labs, students could learn both from the simulation experiments, and also from simply reading the information embedded in the instructions for each lab. To distinguish where the learning occurred, we started each session by having students read a description of osmosis and diffusion. With the osmosis lab, we used several pages from a popular introductory biology text (Freeman, 2002, pp. 77-79). However, this text (and others) contains an inaccuracy in its description of diffusion. Freeman uses an example of billiard balls bouncing off each other and describes diffusion as occurring because the balls bounce off each other more frequently when they are close together, a description that is accurate only when the overall density of particles is higher in one place than another. This is rarely true for biologically relevant diffusion processes, so for the diffusion lab, we substituted a one-page introduction that we wrote by combining all the paragraphs of descriptive text embedded in the diffusion lab workbook. Thus, in both cases, before taking the pretest, the students had just read explanations of the processes being explored, and in the diffusion lab, the students had read exactly the same explanatory text they would read during the course of the lab itself. After reading through the written material (which generally took around $10 \mathrm{~min}$ ), each student took a written pretest (Appendix). The students were then asked to work through the computer-based lab on their own, which generally took 45-60 min. When they finished the lab, we asked them to complete a posttest that was very similar, but not identical, to the pretest. We tested the diffusion lab on 15 students working singly. As part of another question asked in this study (see Discussion), we decided to test the osmosis lab primarily with pairs of students (31 total students) so we could record the conversation between the students as they worked. Regardless of whether participants completed the lab individually or as a pair, all students completed both pretests and posttests individually. Copies of all materials used in the study are available upon request from the lead author at info@simbio.com. 


\section{RESULTS}

\section{Confusions and Misconceptions}

As discussed in the Introduction, several authors have investigated student misconceptions around the topics of diffusion and osmosis. What follows is a list of the most common problems we found among the students sampled for this study, before they used the lab, but after they had read descriptions of diffusion, or osmosis, or both. In parentheses next to each description, an indicator shows whether the misconception applies to diffusion, osmosis, or both.

A) Molecules Have a Directional Motion toward Lower Concentration (Diffusion and Osmosis). Students with this misconception think molecules "want" to move from high to low concentrations, either because of some force, because they "sense" there is a lower concentration area, or through some anthropomorphic desire to move away from high concentrations. For instance, one student stated that, "A molecule in a high concentration area feels the force to equalize the concentration and it moves toward the low concentration." The survey question in Figure 1 also elicited this misconception, when students were asked to draw arrows indicating in which direction molecules would be likely to move in the next instant of time. On our prelab surveys, 11 of 15 students drew a picture similar to that in Figure 1a.

B) Equilibrium Is Static (Diffusion and Osmosis). Many students have trouble with dynamic equilibrium, reasoning that once a system reaches equilibrium, all particular movement stops. For instance, one student stated, "They [the molecules] are no longer moving around and are in stasis, until more water or salt or another kind of reaction would occur."

C) Quantity versus Concentration Is Important (Osmosis). Although students state that osmosis will continue until the "concentrations of water are equal on both sides," when asked to draw this, they often draw unequal concentrations of water. For instance, Figure 2 shows an example of a student's answer to a question in which students are asked to draw equal water concentrations on each side, but instead, they often draw equal numbers of water molecules on each side.

D) Diffusion Happens at the Same Speed Regardless of the Concentration Difference (Diffusion). Most students understand that diffusion will move molecules from high to low concentrations, but often do not realize that the difference in concentration determines how quickly this occurs and rarely know why that is true. For instance, one question on our surveys stated that some dye added to the right side of a fish tank started changing the color of the water in the middle of the tank after $20 \mathrm{~min}$ and asked whether it would take less time, the same time, or more time for the color on the left side to start changing. Five of 15 students thought it would take the same time, with rationales similar to: "I assume it will take the same amount of time to travel the same distance."

E) Diffusion Happens Because Solute Molecules Bump into Each Other More Frequently in Areas of High Solute Concentration and This Causes Them To Spread Out (Diffusion). This misconception is not only common among students, it also appears in examples used by some introductory biology texts (e.g., Freeman, 2002, who otherwise has a fine description of these processes). When asked why diffusion occurs, students show this misconception with statements such as: "A molecule that starts in an area of high concentration will be closely surrounded by other molecules of the same kind. During diffusion, the molecule will separate from those other molecules...."

F) Confusion in Calculating and Comparing Concentrations (Osmosis). Some students were not comfortable with the concept of ratios. In other cases, they did not understand how to calculate a concentration. This was particularly problematic when students were asked to compare concentrations in volumes of two different sizes. One question we used to address this was a picture of a tomato in a jar (question 4 on the osmosis test). The jar had a much higher solute concentration and also a much larger total volume than the tomato. When asked to predict the amount of water in the tomato at equilibrium, many students chose amounts based on the numbers of water or salt molecules in the outside and inside solutions, rather than equalizing the concentrations of solute in both solutions.

G) Increased Molecular Density Is Unrelated to Pressure or Volume (Osmosis). Many students did not translate an increase in molecules in an area into an increase in pressure in that area. We saw this, for instance, in the question in Figure $2 \mathrm{a}$ in which five of 31 students did not realize that changing the number of molecules on one side of the container would also change the pressure and thus the volume, as shown by the position of the plungers.

H) Different Solutes Have Differing Effects on Osmosis (Osmosis). Students with this misconception used only some solutes when calculating concentrations (i.e., salt but not a.

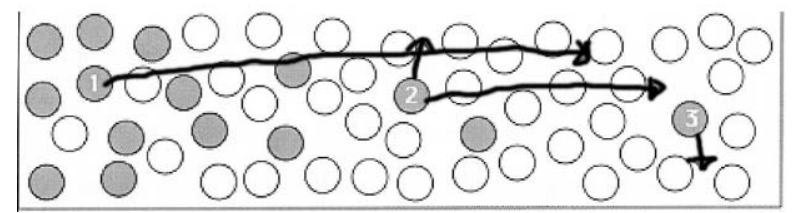

b.

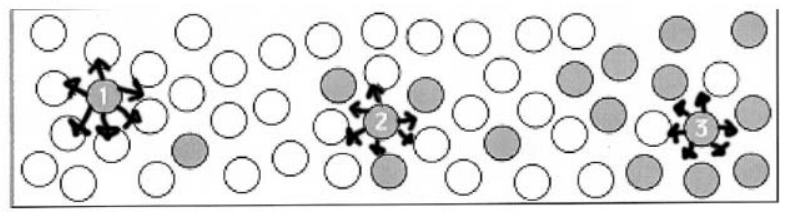

Figure 1. A survey question that elicits misconceptions on directed movement of molecules. This is a typical example of incorrect (a) and correct (b) answers to the question. Before using OsmoBeaker, 11 of 15 students drew arrows (enhanced for clarity) indicating that solutes from high concentration areas would have moved toward lower concentration areas (a), whereas only a few students correctly drew all solutes moving randomly (b). 
a.

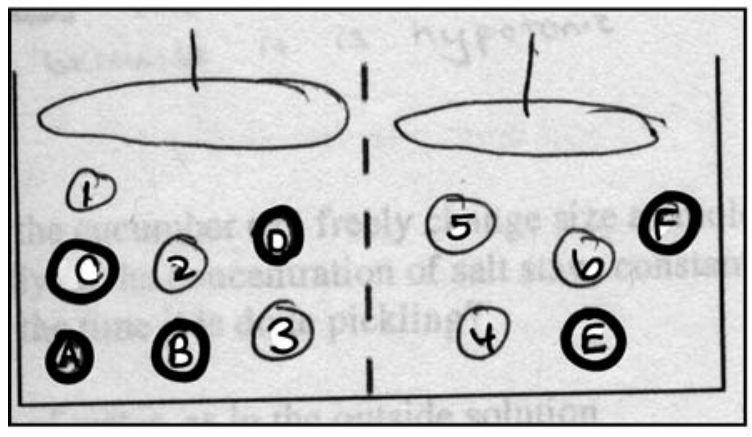

b.

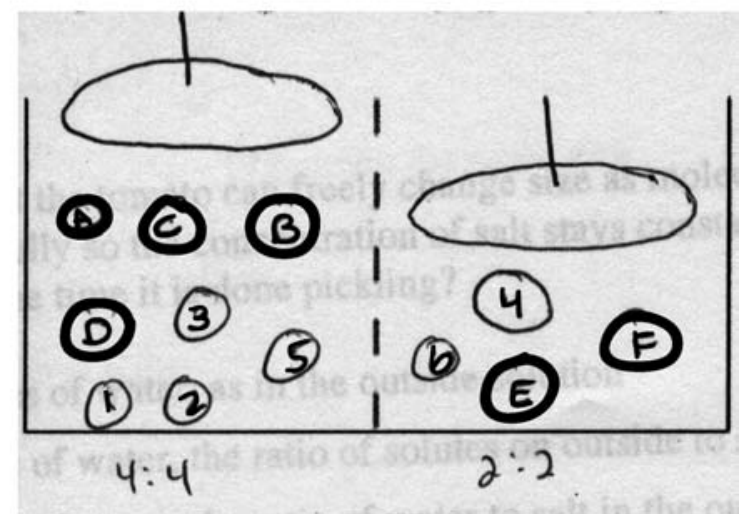

Figure 2. A survey question eliciting misconceptions about solute concentration versus amount, pressure, and dynamic equilibrium. Students are shown the picture of two compartments separated by a membrane that is permeable only to the lighter molecules labeled with numbers (see Appendix). Students are then asked to draw the system when it reaches equilibrium, and a long time after it reaches equilibrium. These are typical examples of incorrect (a) and correct (b) answers. On the pretest, most students drew an equal number, rather than concentration, of light molecules on both sides (a). Only a few students correctly equalized the concentrations (b).

sugar) or assumed that different size particles would have differing effects (i.e., protein adds more to solute concentration than salt).

Table 1 summarizes the proportion of students with each misconception in our study, prior to using one of our labs, from highest to lowest with respect to proportion of student misconceptions.

\section{OsmoBeaker}

As part of this study, we wrote new software, called OsmoBeaker (Meir et al., 2004), which allows users to create and conduct experiments on diffusion and osmosis at the molecular level. OsmoBeaker uses balls of different colors and sizes to represent different types of molecules (Figure 3). The screen contains one or more compartments surrounded by walls, and the molecules bounce within these compartments. One or more walls can be made to move, meaning that when a molecule hits it, the molecule imparts some of its momentum to the wall and the wall is pushed slightly as the molecule bounces off. After trying different configurations of walls, we settled on a single compartment for most of the experiments in the diffusion lab (Figure 3) and two compartments with a movable wall between them for the osmosis experiments (Figure 4) (similar to one of the simulations in the Molecular Workbench software from the Concord Consortium [Berenfeld et al., 2004]). Students are given three available tools to conduct experiments: 1) they can change the initial concentrations of each molecule in each compartment, 2) they can define a wall as either permeable or impermeable to the different molecules, or 3) they can manually slide a movable wall and then release it from its new position (an action that can be used to test equilibrium).

Using this setup and tools, we wrote two labs, one focusing on diffusion and the other on osmosis. Each lab guides students through a series experiments specifically designed to elicit likely student misconceptions drawn from the list above. The diffusion lab focuses on the misconceptions on Directed Motion, Static Equilibrium, Diffusion Speed, and Molecule Bumping (A, B, D, E), while the osmosis lab focuses on Static Equilibrium, Quantity versus Concentration, Concentration Calculations, Pressure, and Multiple Solutes (B, C, F, G, H). The labs are heavily scaffolded, with explicit step-bystep instructions describing the experiments that students should perform throughout the lab.

In the diffusion lab, the experiments are built around the question of whether nerve cells could use diffusion to transport peptides that are created in the cell body down to the tip of the axon. The lab starts with an example of a cell without a permeable membrane, and then adds channels to the membrane to give a visual clue to describe diffusion. The next section of the lab has three axons with lengths of 15,30 , and $60 \mu \mathrm{m}$, and a cell body on the left producing peptides (Figure 3). Students time how long it takes for the first peptide to reach the other end of each axon, and then plot these data to see that the diffusion time increases exponentially with distance. Underlying all these exercises is an emphasis on trying to get students to observe the

Table 1. Frequency of confusions and misconceptions regarding diffusion and osmosis ${ }^{a}$

\begin{tabular}{ll}
\hline \multicolumn{1}{c}{ Misconception } & $\begin{array}{c}\text { Percentage of students } \\
\text { initially demonstrating } \\
\text { this misconception }\end{array}$ \\
\hline Static Equilibrium [B] & \\
Quantity versus Concentration [C] & $80.0 \%(\mathrm{n}=15) 12 / 15$ \\
Directed Motion [A] & $76.7 \%(\mathrm{n}=43) 33 / 43$ \\
Concentration Calculations [F] & $73.3 \%(\mathrm{n}=15) 11 / 15$ \\
Diffusion Speed [D] & $58.1 \%(\mathrm{n}=31) 18 / 31$ \\
Multiple Solutes [H] & $53.5 \%(\mathrm{n}=15) 8 / 15$ \\
Pressure [G] & $46.9 \%(\mathrm{n}=32) 15 / 32$ \\
Molecule Bumping [E] & $42.2 \%(\mathrm{n}=45) 19 / 45$ \\
& $26.7 \%(\mathrm{n}=15) 4 / 15$
\end{tabular}

${ }^{a}$ The first column lists the misconceptions using the letters from the list above. The second column indicates the number of students who answered one or more questions incorrectly on the pretests about that misconception. 

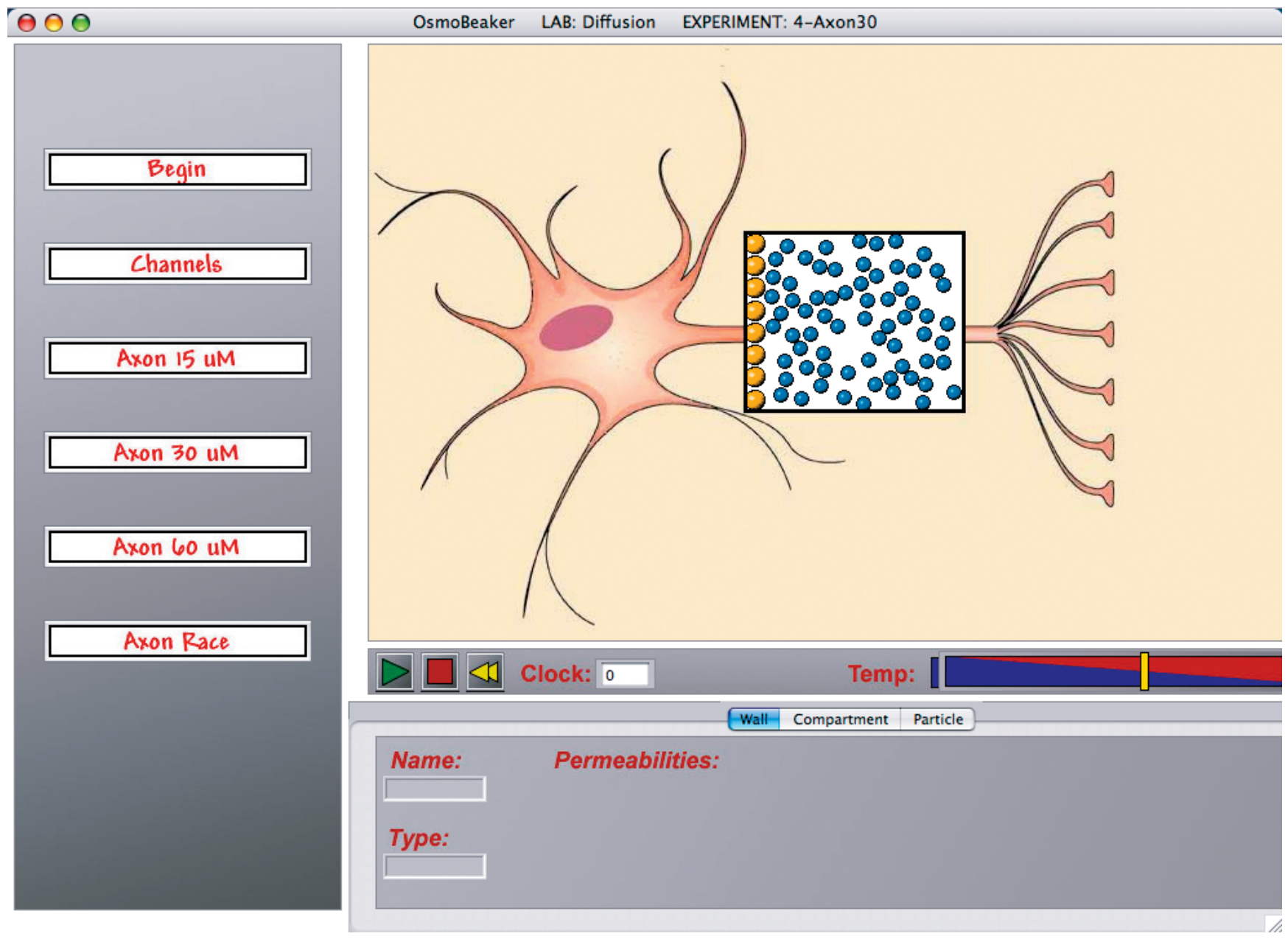

Figure 3. One of the three axon-length experiments in the diffusion lab. The cell body on the left is producing peptides (large yellow balls) that must reach the tip of the axon on the right, diffusing through water molecules (small blue balls) along the way. Students run the model and stop it when the first peptide reaches the right-hand tip of the axon, timing how long it takes for diffusion to move materials the length of this axon. They repeat this experiment in axons one-half the size and $2 \times$ the size of this one. These data lead students to not only confront the Diffusion Speed misconception, but more fundamentally, to confront the Directed Motion misconception.

randomness of the motion both before and after equilibrium is reached. The final exercise is an experiment designed to address the Directed Motion and Molecule Bumping misconceptions (A, E). During this scenario, called Axon Race, students are presented with a high initial concentration of peptides on the left edge and only a single peptide on the right edge. One peptide on the left is marked with a dot, and a peptide on the right is marked with $\mathrm{a}+$. We then ask students which of these two peptides will, on average, reach the other side first. Students with a misconception will predict that the peptide on the left will reach the right side faster than the peptide on the right reaches the left side. Students run this scenario a number of times, recording the average crossing time of each marked peptide. On average, of course, students discover that both marked peptides reach their opposite wall in roughly the same amount of time.

In the osmosis lab, the experiments are written around the scenario that the user is giving intravenous (IV) fluids to a patient in a hospital, and students must determine the concentration of solutes in the IV fluid so that the patient's red blood cells do not shrink or expand. The lab has two compartments, the left one, called the red blood cell (RBC), and the right one, an extracellular compartment containing IV fluid (Figure 4). Separating these two compartments is a cell membrane. When the lab begins, there is an impermeable membrane to show that pressure results from molecules hitting the membrane. In the first experiment, the IV fluid contains only water, while the RBC has both water and salt. Students open water channels in the membrane and watch the cell blow up. The next experiment has students add salt to the IV fluid and see what salt concentration no longer causes the cell to expand. The next experiment shows a cell with both salt and sugar molecules; however, students are allowed to add only salt to the IV fluid. This addresses the Multiple Solutes misconception. We also address the Quantity versus Concentration misconception (C) in this experiment by making the RBC a different size than the IV fluid compartment (Figure 4). Students with this misconception will match the solute amounts rather than solute concentrations and see the cell 
a.

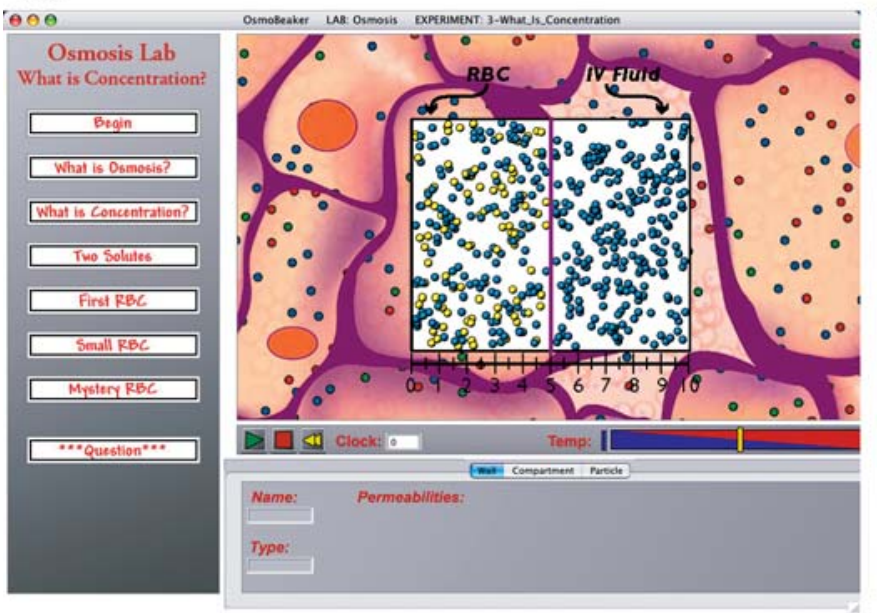

b.

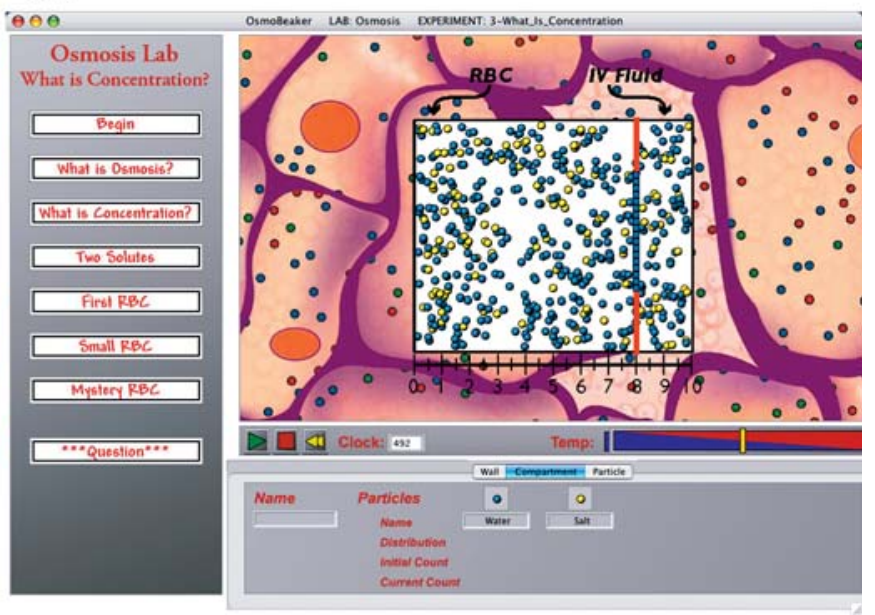

Figure 4. One experiment from the osmosis lab. A red blood cell is on the left, and an extracellular compartment filled with IV fluid is on the right. One of the cell's membranes appears down the middle and can move in response to pressure. (a) Before running the simulation, students are asked to change the concentration of salt in the IV fluid so the cell does not change size over time. (b) Students then run the model. In this case, the IV fluid was too dilute, causing the cell to expand over time.

expand instead of staying the same size. The final two experiments use RBCs containing salt, sugar, and hemoglobin, the first experiment showing an RBC that is equal in size to the IV fluid compartment, the second with an RBC much smaller than the IV fluid compartment. In all sections of the lab, we ask students to calculate the concentration of solutes needed before actually trying the experiment, and to describe and justify their results afterward. Thus students make many calculations of concentrations throughout the lab, and learn to match concentrations instead of quantities.

\section{Diffusion Laboratory Results}

As shown in Table 2, posttest scores demonstrate that at least some students improved on each targeted misconception relative to the pretest. However, our results also suggest that the lab was more effective at helping students overcome some misconceptions than others. The most significant posttest gains were on the Directed Motion misconception (A). Figure 1 shows question 3 from our diffusion test, on which almost all students improved after performing the experiment. Of the 11 students who drew a picture similar to that shown in Figure 1a on the pretest, three drew the correct picture (Figure 1b) after the lab, while six drew a picture that was a hybrid between the two pictures shown in Figure 1. In either of those cases, students show an understanding that molecules are moving randomly in all directions, not just in the direction in which diffusion is taking place. The diffusion lab also appears successful at helping students overcome misconceptions on Static Equilibrium, Diffusion Speed, and Molecule Bumping, (B, D, E), though less successful than for the Directed Motion misconception. One-third to one-half of the students initially demonstrating each of the latter misconceptions show improved understanding after completing the lab.

Overall, 13 out of 15 students we tested improved after performing our diffusion lab, with the remaining two showing no change. The students had an average pretest score of
$4.2(\mathrm{SD}=2.5)$ (out of a possible 10$)$ and an average posttest score of $6.7(\mathrm{SD}=2.3)(60$ percent improvement, $P<.001)$.

\section{Osmosis Laboratory Results}

As with the diffusion lab, we saw improvements on our osmosis posttest relative to the pretest for most questions we asked (Table 3), but found the largest improvements on the Quantity versus Concentration, Concentration Calculations, and Multiple Solutes misconceptions (C, F, H). Overall, 23 of 31 students we tested improved, while four did worse, and four showed no change. Students had an average pretest score of 10.0 (out of a possible 18; SD =3.8) and an average posttest score of $12.2(\mathrm{SD}=4.0 ; 22$ percent improvement, $P<.001)$.

Table 2. Improvement observed with students performing the diffusion laboratory experiment in OsmoBeaker ${ }^{a}$

\begin{tabular}{lcc}
\hline $\begin{array}{c}\text { Question on diffusion test } \\
\text { [misconception] }\end{array}$ & $\begin{array}{c}\text { Pretest }(\mathrm{n}=15) \\
\text { number correct } \\
(\% \text { correct })\end{array}$ & $\begin{array}{r}\text { Posttest }(\mathrm{n}=15) \\
\text { number correct } \\
(\% \text { correct) }\end{array}$ \\
\hline 2. [Static Equilibrium] & $3(20 \%)$ & $7(47 \%)$ \\
3. [Directed Motion] & $4(27 \%)$ & $13(87 \%)$ \\
4b. [Diffusion Speed] & $7(47 \%)$ & $11(73 \%)$ \\
5b. [Directed Motion, & $9(60 \%)$ & $13(87 \%)$ \\
$\quad$ Static Equilibrium, & & $14(93 \%)$ \\
6a. [Diffusion Speed] & $13(87 \%)$ & $12(80 \%)$ \\
6b. [Directed Motion, & $7(47 \%)$ & \\
$\quad$ Molecule Bumping] & & \\
\hline
\end{tabular}

${ }^{a}$ The question numbers refer to questions in the Diffusion Survey that appears in the Appendix. The brackets beside each question number indicate the misconception the question was testing using abbreviations from the Confusions and Misconceptions section. In a few questions $(1,5 b$, and $6 a)$, we found one example of a student performing worse on the posttest than on the pretest (no question showed more than one student's responses declining after the lab). 
Table 3. Improvement observed with students performing the osmosis lab in OsmoBeaker $^{a}$

\begin{tabular}{|c|c|c|}
\hline $\begin{array}{l}\text { Question on osmosis test } \\
\text { [misconception] }\end{array}$ & $\begin{array}{c}\text { Pretest } \\
\text { number correct } \\
\text { (\% correct) }\end{array}$ & $\begin{array}{c}\text { Posttest } \\
\text { number correct } \\
\text { (\% correct) }\end{array}$ \\
\hline 1b. [Directed Motion] & $2 / 31(6 \%)$ & $5 / 31(16 \%)$ \\
\hline 1c. [Static Equilibrium] & $22 / 31(71 \%)$ & $23 / 31(74 \%)$ \\
\hline $\begin{array}{l}\text { 1d. [Quantity versus } \\
\text { Concentration] }\end{array}$ & $9 / 31(29 \%)$ & $17 / 31(55 \%)$ \\
\hline 2. [Multiple Solutes] & $17 / 31(55 \%)$ & $27 / 31(87 \%)$ \\
\hline 3a. [Static Equilibrium] & $11 / 31(35 \%)$ & $11 / 31(35 \%)$ \\
\hline 3b. [Pressure] & $21 / 31(68 \%)$ & $24 / 31(77 \%)$ \\
\hline $\begin{array}{l}\text { 3c. [Quantity versus } \\
\text { Concentration] }\end{array}$ & $6 / 31(19 \%)$ & $13 / 31(42 \%)$ \\
\hline $\begin{array}{l}\text { 4a. and } 4 \mathrm{~b} . \\
\text { [Quantity } \\
\text { versus Concentration, } \\
\text { Concentration } \\
\text { Calculations] }\end{array}$ & $21 / 31(68 \%)$ & $23 / 31(74 \%)$ \\
\hline $\begin{array}{l}\text { 4c. and } 4 \mathrm{~d} \text {. } \\
\text { [Concentration } \\
\text { Calculations] }\end{array}$ & $13 / 31(42 \%)$ & $19 / 31(61 \%)$ \\
\hline $\begin{array}{l}\text { 5a.-5c. } \\
\text { [Concentration } \\
\text { Calculations, } \\
\text { Multiple Solutes] }\end{array}$ & $12 / 31(39 \%)$ & $26 / 31(84 \%)$ \\
\hline 1a. $[\mathrm{N} / \mathrm{A}]$ & $26 / 43(60 \%)$ & $32 / 43(74 \%)$ \\
\hline 1e. $[\mathrm{N} / \mathrm{A}]$ & $29 / 43(67 \%)$ & $31 / 43(72 \%)$ \\
\hline
\end{tabular}

${ }^{a}$ See Table 2 for an explanation of data.

Question 3 (Figure 2), which asked students to give an initial distribution of molecules within a U-shaped tube and asked them to draw a picture of what it would look like at equilibrium and a long time after equilibrium, was the hardest of the questions and one of the most informative. We scored this question for three things: 1) Did the student make the concentrations of molecules equal on both sides? 2) Did the student indicate that this would generate a pressure as shown by moving the left plunger higher than the right? 3) Did the student recognize that even at equilibrium, molecules would keep moving back and forth between the two sides? We saw the most improvement on concentration. Many students made the number of molecules equal on both sides, rather than the concentration, and some students were more confused and left all molecules in their initial positions, or even increased the concentration difference. Of the 25 students who answered the questions incorrectly on the pretest, seven realized the correct concentrations on the posttest. Learning on this same topic is also seen in question 1d, a justified true/ false question on concentration versus number of molecules, on which eight of 22 students improved.

In contrast, students did not seem to improve their understanding of the dynamic equilibrium of molecular concentrations. In the U-tube question in Figure 2, each molecule had a number or letter inside of it. We asked students to draw a third picture of what the system looked like a long time after equilibrium (see Appendix). Students who understood that the equilibrium was dynamic should have left the same number of molecules on each side, but switched some of the numbered molecules between the two sides to show that they continued to move even though the concentrations remained unchanged. Although 11 students answered correctly on the pretest, no students changed their answer here between the pretest and posttest. This lack of learning was confirmed by question 1c, a justified true/false question on the same topic, on which only one student improved between pretests and posttests.

\section{Why Did Students Change Their Answers between Pretests and Posttests?}

To better see how students' understanding of the core concepts changed through the osmosis lab, we asked each student to compare their pretest and posttests and explain any differences in their answers. Most of the differences were on questions on which we saw gains in understanding, which gives us insight into how the program aids students in overcoming misconceptions. We analyzed these interviews for three sets of misconceptions, the Multiple Solutes misconception together with Concentration Calculations problems, the Quantity versus Concentration and Pressure misconceptions, and the Static Equilibrium misconception. In each but the last, student interviews provide clues to how their thinking had changed after doing the lab, while for the Static Equilibrium misconception, the interviews give us clues for where we still need to improve the program.

\section{Multiple Solutes and Concentration Calculations}

Many of the students noted in their interviews that after doing the lab, they realized total solute concentration is important for osmosis rather than the concentrations of individual solutes, indicating improvements in the Multiple Solutes misconception (and also often on Concentration Calculations). For example, student A changed her answer to question 2, concerning the concentration of solute needed to make an isotonic solution, and expressed her reason as follows: "Before I didn't understand what you'd do if you had two different solute concentrations, but it doesn't matter; like the individual concentrations, it's just the overall concentration of the individual solutes, so I changed to the total number of solute, or the total concentration of solute."

This student clearly now understands that solutes are equivalent in osmosis, when she didn't before. Student J had a similar comment on changing question 2, and also commented on changing question 5, concerning cell size changes in different cells with the same overall but different individual solute concentrations: "Once again, I just did the total, and that's 20 and that's 20, so regardless of how the breakdown is [in different solute molecules], it's still 20, so they both expand, since the solute concentration is only five in both, so they expand at the same rate."

Student $G$ also changed her answer on question 2 and said: "It's to make an isotonic solution. It has to have the same concentration of solvent, of solute, as the cell, so that's what I did. That one [pretest] I didn't know what I was doing."

This student also now understands that solutes are equivalent, but expressed greater confusion on the pretest than student A. It is hard to attribute this confusion to lack of exposure to osmosis though, because she reported that her high school advanced placement biology class spent two lectures and a 3-h lab on osmosis, and her current college course discussed it during lecture. It thus seems likely that 
she previously had been taught this concept, but had not internalized the idea (or how to put it into practice).

From these interviews, it seems that the repeated practice in the lab that asks students to calculate isotonic solutions with multiple solutes is effective. By the end of the lab, students are comfortable adding up the concentrations of all the solutes and making inferences based on those total concentrations, something that they were often not comfortable with beforehand.

\section{Quantity versus Concentration and Pressure Misconceptions}

Quite a few students improved on the Quantity versus Concentration misconception as shown with two questions, the justified true/false question 1d (it is false that osmotic equilibrium depends on number of solvent molecules), and the balls-and-plungers question (question 3) in Figure 2. A few students also showed improvement in the Pressure misconception on that question. The posttest interviews and written descriptions of their answers indicated that some of these changes were the result of genuinely overcoming misconceptions. But students who corrected their answer only on the true/false question may have simply learned to use better terminology, without an increased understanding of the concepts.

Student I clearly improved her understanding of both Quantity versus Concentration and Pressure misconceptions. This student created a drawing similar to that of Figure 2a on the pretest, with plungers at equal heights and equal numbers of water molecules on either side rather than equal concentrations. Despite that, the written explanation accompanying her drawing read, "The permeable molecules transfer so that they are equal in concentration." On the posttest, her drawing was correct, with an explanation reading, "The ratios will be equal, and the left plunger will be higher than the right because there are more molecules and more pressure." In the interview, she said, "The ratios are equal now. I really didn't know that before, I just kind of took a random guess." Before the lab, although she could say that the concentrations should be equal, she equated concentration with number. After the lab, she equated concentration with a ratio of solute to solvent, a conceptual improvement, and correctly projected that the difference in molecule number will lead to a difference in pressure.

Student L also improved her understanding of concentration between the pretest and posttest, although she still was not able to produce an accurate posttest drawing. On the pretest, she moved one solute molecule from the left to the right and one solvent from the right to the left, so that there were equal numbers of both solute and solvent on both sides. On the posttest, she left all the solute on the same side, while still moving one of the solvent molecules from right to left (she should have moved two solvent molecules to make concentrations equal, but this was at least in the correct direction). The student explained the change as:

Before, I thought I knew the equilibrium was crossing over, to have an equal concentration gradient, but I didn't understand what would happen with the black cells [solutes] because I thought that it would have to be equal pressure on either side, but what I now realize is that ... you can still have more molecules on one side than on the other as long as there's an equal concentration, so what I decided was that the water would diffuse across, at least one would, so that there was an even three water molecules on either side but there would still be four filled-in molecules and then two on here, so there would be more pressure on the left than on the right.

This student now understands that equal concentrations does not mean the same number of each type of molecule inside and outside the cell. Furthermore, she now sees that the pressure can be different on the two sides at equilibrium, because, as with student I, student L also correctly drew a difference in plunger height on the posttest after missing that on the pretest.

Another student, student A, drew pictures similar to those in Figures $2 \mathrm{a}, \mathrm{b}$ on the pretest and posttest, and on the posttest wrote: “... the open circle molecules [solvent] will enter the membrane and attempt to create equal ratios of concentration on both sides," but on the pretest she wrote only: "... open circles would move from the area of higher concentration to an area of lower concentration" without mentioning equalizing concentrations or ratios. However, this student did not seem that confident in her new understanding in the follow-up interview, as evident by the following conversation:

Researcher: You changed the position of the plungers, so can you tell me about that?

Student: Well, since the membrane only lets in the open circle molecules, then more things are going to be rushing in, so I figured the plunger would be higher, I didn't really know how to balance them out, though I figured they'd try to be establishing the same ratio on both sides but I didn't really get it. [Be]cause I think initially I thought maybe like one of these would rush in to balance the three and three, but I didn't know what that would do in relation to the dark ones.

Researcher: So over here you drew it four and four and two and two. Can you tell me about that?

Student: I guess, I don't know, I was trying to create a similar ratio but I don't think those are the same ratio.

Researcher: Four out of four and two out of two are not the same ratio?

Student: I guess they are [be]cause it's like one to one, so, I don't know.

While the student is not confident in describing the new answer, both the interview and written explanations suggest that she is approaching a better conceptual understanding on both Quantity versus Concentration and Pressure misconceptions, because she is now looking at ratios of solute and solvent, and drawing the equilibrium with a pressure difference between the two sides.

These students showed improvement on both ball-andplunger and true/false test questions (question 1d), and their subsequent explanations lent support to the hypothesis that these changes reflected improved conceptual understanding. Several other students, though, correctly changed their answer to the true/false question, but did not change their balls-and-plungers drawing. These could be cases of partially overcoming the misconceptions, or they could be students who simply realized the correct vocabulary to use during the course of the lab. For example, student $G$ described why she 
changed her answer on question 1d as follows: "It doesn't depend on the water concentration, or the number of solvent molecules in the system, on either side of the membrane. It's based on the concentration of the solute compared to, like, compared with each other."

This student actually had the molecules drawn correctly on question 3 in both the pretest and posttest, but wasn't able to translate that into talking about concentrations rather than numbers on the true/false question. Even in this answer, this student still seems to harbor confusion about water concentration and solute concentration being different things rather than two ways of expressing the same quantity. Student $\mathrm{N}$ similarly changed her true/false answer without changing her (incorrect) drawing in the balls-and-plungers question, saying, "The important part was the water concentration, not, like, the number of molecules. It doesn't really matter, it's just about the concentration."

Student K actually did worse on the posttest balls-andplungers question, writing that she was not sure what to do, but also changed her answer to question $1 \mathrm{~d}$, saying she did so, "Because when we were doing the stuff on your laptop [the osmosis lab], it didn't have anything to do with amount really, but more with concentration."

In these cases, it seems that either the learning that took place did not transfer to the balls-and-plungers question (which had a different configuration than the simulations in the lab) or that students just learned to say "concentration" without any increased understanding. This makes us trust our answers from the questions that ask for drawings or calculations much more than those from the true/ false questions, even with accompanying written justifications.

Overall, the interviews confirm that students' answers on the balls-and-plungers question generally reflect their understanding of several concepts, while our justified true/false questions on those topics are not as good an indicator of conceptual understanding. Students' verbal comparisons of their prelab and postlab answers, however, do suggest that some students make real conceptual gains on Quantity versus Concentration and Pressure misconceptions through performing the lab.

\section{Static Equilibrium}

We were disappointed that the osmosis lab did not seem to help students overcome the Static Equilibrium misconception. Along with the two misconceptions discussed above, we also scored the balls-and-plungers question (question 3) for this misconception, as well as the justified true/false question 1c. Most students did not change their answers on either, so we did not ask them about it in the interviews, but student L actually made a better drawing on the pretest than the posttest, and her interview may shed some light on why the lab did not help overcome this misconception.

Student: Down here [posttest] I just said that it [the molecules] would probably just stay the same. There would still be more pressure on the left than on the right and that, once its reached equilibrium, it will pretty much stay there.

Researcher: And here [pretest] you had moved more water molecules over onto the left.
Student: Yes, I thought [on pretest] that there'd still be a lot of fluctuation after equilibrium, and I didn't know exactly how to show that, but that there'd still be a lot of moving back and forth, in that it would slowly diffuse away from equilibrium.

The student's response suggests that this student was unable to see the molecules crossing the membrane during equilibrium in the osmosis lab. A yes/no question in the lab itself asks whether water molecules are still crossing the membrane at equilibrium, and this student answered "No." Thus, it may be that the simulation itself was inadequate in making apparent the movement of molecules across the membrane during equilibrium. However, on another question (question 4), this student also made a general comment about how the lab helped her overcome the Quantity versus Concentration misconception: "Well, in the lab there were a lot of questions that, like, at the end once you had sort of gotten comfortable with how to make them equal you realized that it wasn't the amount, like, of molecules or the types of molecules, it was just the ratios, and that's what would make it equal, so you could see like, a lot of different movement and that's what, so sort of, like, the red blood cell question would helped me out with that."

This indicates that it was not that this student was incapable of learning from the lab, but rather, the lab did a poor job addressing that one particular misconception (Static Equilibrium).

\section{Are the Laboratories More Effective for Some Classes of Students?}

While observing students performing the laboratories, we had the impression that they were more effective for some types of students than others. For instance, a few students came into our labs already understanding diffusion and osmosis quite well. These students had high scores on the pretest, and therefore had little scope for improvement on the posttest. Other students struggled with concepts that the lab assumed they would already be comfortable with, such as calculating ratios. The bulk of the students came to us with many misconceptions, but all the fundamental skills needed to easily complete the laboratories. We hypothesized that the latter group of students would benefit most from the computer labs, while the best-prepared and worst-prepared students would show smaller gains.

We could not find evidence for this hypothesis in the data, however. We split the students into three equal-sized groups based on their pretest scores and examined the amount of improvement for each group on the posttest. The bottom one-third improved the most (average improvement $=3.3$ ), the middle group improved less (average improvement = 1.8 ), and the top one-third improved the least (average improvement $=1.6$ ). Because the bottom one-third had the most scope for improvement (they answered more questions incorrectly to begin with, and so they could improve on more questions), this result is not very informative. We also tried correlating improvements with other measures that we collected from the students, such as grade point average, grade in their most recent biology course, or whether they had conducted a wet osmosis lab. None of these showed any significant correlations (data not shown). Students who had never studied osmosis in a class improved more than those 
who had studied osmosis a little $(\leq 1 \mathrm{~h})$ or a lot $(>1 \mathrm{~h})$, but this was also statistically insignificant $(P=.06)$. With the measures we used here, it does not appear that we can identify differences in which students who might benefit most from the labs we wrote.

\section{DISCUSSION}

The misconceptions found in our research mostly overlap those found by other authors. For instance, Odom (1995) finds that 29 percent of college biology students think molecules "want to" move to lower concentrations (similar to our Directed Movement misconception) and 27 percent think that molecules "crowded into one area" cause diffusion (as in our Molecule Bumping misconception). Zuckerman (1994) found that advanced high school students often have misconceptions similar to our Directed Motion, Quantity versus Concentration, and Dynamic Equilibrium misconceptions. We also interviewed five college biology teachers at the National Association of Biology Teachers annual meeting in the fall of 2003 and found that they mentioned many of the same problems among their own students (data not shown). Thus our list of eight points of misconception and confusion serves as a worthwhile challenge for new teaching tools to overcome.

Our diffusion lab is particularly effective at overcoming the Directed Motion misconception. The experiments seemed to help students understand how random motion at a small scale can lead to organized behavior at a larger scale. Based on our observations of students utilizing the lab and interviews conducted after they completed the posttest (data not shown), we believe this improvement occurs for two reasons. First, throughout the lab, students observe molecules moving randomly, and just seeing the process dynamically helps students reconceptualize how molecular movement occurs. Second, we explicitly ask students to watch individual molecules at several points in the lab. For example, early in the diffusion lab, students are asked to notice any differences in the behavior of particles when an initially impermeable membrane is made permeable. In another example, as students observe individual particles in the Axon Race experiment described above, the students are likewise compelled to face their potential misconception. We also found some improvement on the Static Equilibrium and Diffusion Speed misconceptions. There is a section of the lab devoted to how concentration affects diffusion speed, and while observing students perform the lab, we noticed several times how students seemingly changed their understanding while doing those exercises.

Our osmosis lab was most effective at addressing misconceptions on Quantity versus Concentration and on Multiple Solutes. The osmosis lab has a section in which students are asked to calculate the ratios of solute to water at equilibrium and see that it is the same in both compartments. They must also think in terms of concentrations of solute at many other points in the lab. By the end, most students who came in confused about how concentrations of solutes drive osmosis are much more facile at solving problems in terms of solute concentration. This is shown most powerfully in the balls-andplungers test question (Figure 2), a different configuration of compartments than that used in the lab. Thus for students to change their answer on this question between pretest and posttest requires a transfer of knowledge from one presentation to the other, and the fact that seven of 25 students corrected their answers on the posttest is strong evidence that real learning took place. The interviews of students who changed their answers on this question seem to confirm that conclusion, although changes in answers on the justified true/ false question on the same topic may be misleading.

Our labs were disappointing in their results for two of the misconceptions, Dynamic Equilibrium and Pressure (B, G). Although the diffusion lab did help some students understand dynamic equilibrium, we explicitly focused on this concept in the osmosis lab, yet saw no improvement there. The osmosis lab exercises on equilibrium focus on pressure rather than concentration. Students are asked to test whether the cell size is at an equilibrium by pushing on the cell membrane and seeing if it comes back to its original position. Another question explicitly asks, "Once the membrane has reached an equilibrium position, is water still flowing across the membrane (Yes or No)?" However, students are never asked to perform exercises in which they must watch individual molecules or make any kinds of predictions that would contradict a misconception that molecules remain in the same compartment once equilibrium is reached. As seen in the interview with student $\mathrm{L}$, it appears just watching the molecules continue to move at equilibrium, and exercises on pressure equilibrium, are not enough to overcome student misconceptions on how a system can be at equilibrium overall while individual molecules continue to move. It is possible that had we asked questions about whether systems at equilibrium are frozen in place, we would have seen improvement as students recalled their experiments pushing the membrane to test for equilibrium. Similarly, although students see that molecular density leads to changes in cell size (through pressure changes), they are unable to transfer these observations to changes in pressure on our test questions. In both these cases, we think it would be useful to try adding additional experiments that more explicitly confront these misconceptions, and will try that in a future version of the labs.

Our work also emphasizes the key role that the written instructions accompanying the simulations play in promoting learning. This is seen, for instance, in the Directed Motion misconception. Although the molecules are moving randomly, with basically the same dynamics in both the diffusion and osmosis simulations, the workbook in the diffusion lab contains experiments and questions that emphasize random motion, whereas the osmosis lab does not focus on this at all. Not surprisingly, many students who performed the diffusion lab experiments improved on this misconception, whereas very few improved on this concept after performing the osmosis lab experiments. As we designed and refined each lab, we ran into many cases in which, although students had the tools to see and experiment with a particular concept in the simulation, they did not actually explore that concept until we explicitly wrote instructions to guide them through that exploration. This shows that simply presenting a simulation environment to students is not enough to promote learning. The scaffolding provided by our workbooks or similar instructional materials is critically important.

Our study also falters in discerning differences between subclasses of students. While we thought we would see certain groups of students (for instance, those coming in with intermediate levels of preparation) improve more than others, we did not find any clear evidence for such 
differences. This may be in part because our student volunteers were very diverse and self-selected. While we deliberately went for diverse ability levels in this study by recruiting from 11 widely varying campuses, in the future it would be valuable to focus on fewer campuses and to explicitly try to increase the number of students with distinct ability levels, preparation in the topic, and so on. In that way, we can better differentiate ways in which this teaching technique is most effective.

A final improvement we plan for the next version of the software is to make the laboratories more complex and openended. This is complicated by the inherent variability in the simulations. Even with the current, highly tuned, directed experiments, a percentage of students will get the wrong answer in a couple of the exercises because there are too few molecules in the simulation for it to show the real pattern 100 percent of the time. As we add more open-ended experiments, we may need to introduce biases into the modeling engine that mimic the effect of having millions of molecules (which is currently not possible to model quickly enough).

We used very similar questions for both pretests and posttests in order to make comparison easier. Our impression was that some students remembered their answers from the pretest and filled in the same answers on the posttest without giving much thought to any learning they may have incurred during the lab. Thus we think that the measured improvements shown in the tables are likely conservative, and that more in-depth probing of the students' conceptual knowledge might show greater improvement.

\section{CONCLUSIONS}

Here we have shown that inquiry-based simulated labs at a molecular level help students overcome several common misconceptions about diffusion and osmosis. These improvements are attributable to the simulated experiments. Most students in our study had previously received a lecture in their introductory biology class on osmosis, many had performed a wet lab on osmosis, and all had read several pages of material on diffusion, osmosis, or both immediately prior to taking our pretest. Thus, the improvements observed after the computer laboratories are above and beyond what students learn by reading or listening to material on the topic. We expect that similar simulations (e.g., Berenfeld et al., 2004) accompanied by labs with similar scaffolding would also show similar improvements. This study does not address the issue of whether other active-learning strategies would lead to equivalent improvements in understanding, itself an interesting but separate question. For instance, paper-and-pencil problem sets might have been just as effective at overcoming the Multiple Solutes misconception, because it is just a matter of adding up different solutes rather than treating them separately. Intuitively, we expect that some of the other misconceptions such as Directed Motion, Quantity versus Concentration, Pressure, and Dynamic Equilibrium are best addressed with simulations because the visual feedback and dynamics are directly relevant to these dynamic processes.

OsmoBeaker was designed as part of a larger project to try to use simulations to assess students' understanding of biological concepts. Our ultimate goal is to have the computer "observe" the experiments that students perform and deduce from their actions what misconceptions they have. This would allow the software to change the presentation of the laboratory to suit each individual student's needs; for instance, presenting a student who has trouble calculating concentrations with more practice on that while skipping ahead to harder concepts and experiments for students who appear to understand the basic mechanism of osmosis. We believe our results here show that OsmoBeaker is a good platform on which to build trials of that technology.

Regardless of the outcome of the larger aims for this study, the approach taken in OsmoBeaker toward teaching concepts normally invisible to the naked eye appears to be effective. By conducting their own experiments at the molecular level, many students were able to overcome molecular-level misconceptions and gain a better understanding of diffusion and osmosis. This approach should extend to other areas, such as neurobiology and biochemistry, which share a similar disconnect between the level at which a phenomenon can be observed in real life and the level at which it actually takes place.

\section{ACKNOWLEDGMENTS}

This research was supported in part by grant 0230740 from the U.S. National Science Foundation, and in part by SimBiotic Software. Lance Rogeux helped with some of the initial data collection. We thank Susan Yoon, Ben Snyder, June Zuckerman, and Mary-Anne Ramirez for helpful discussions, and Christopher Watters and two anonymous reviewers for their thoughtful comments.

\section{REFERENCES}

Berenfeld, B., Damelin, D., Pallant, A., Tinker, B., Tinker, R., and Xie, Q. (2004). Molecular Workbench. The Concord Consortium. http:// www.concord.org (accessed 15 September 2004).

Bialek, W., and Botstein, D. (2004). Introductory science and mathematics education for 21 st-century biologists. Science 303(5659), 788-790.

Freeman, S. (2002). Biological Science. New Jersey: Prentice Hall.

Gibbons, N.J., Evans, C., Payne, A., Shah, K., and Griffin, D. (2004). Computer simulations improve university instructional laboratories. Cell Biol. Educ. 3, 263-269.

Meir, E., Stal, D., and Maruca, S. (2004). OsmoBeaker 1.0. Ithaca, NY: SimBiotic Software.

Odom, A.L. (1995). Secondary \& college biology students' misconceptions about diffusion \& osmosis. Am. Biol. Teach. 57, 409-415.

Roschelle, J.M., Pea, R.D., Hoadley, C.M., Gordin, D.N., and Means, B.M. (2000). Changing how and what children learn in school with computer-based technology. Child. Comput. Technol. 10(2), 76-101.

Sanger, M., Brecheisen, D.M., and Hynek, B.M. (2001). Can computer animations affect college biology students' conceptions about diffusion \& osmosis? Am. Biol. Teach. 63, 104-107.

Zuckerman, J.T. (1994). Problem solvers' conceptions about osmosis. Am. Biol. Teach. 56, 22-25. 


\section{Appendix}

The posttest we used for each laboratory is reproduced here. The pretests were substantially similar, with very small changes in some questions to try and prevent students from simply regurgitating their answers from one test to the other. (From our observations, we don't think these small changes were particularly effective at that task.)

\section{Diffusion Survey}

1a. In your own words, define diffusion.

1b. In your own words, describe what is happening to an individual molecule that starts in an area of high concentration.

1c. Does diffusion require a membrane in between the high and low concentrations?

Yes

No

2. You have a baking sheet filled with water and dissolve some powdered sugar on one side. You let the sheet sit for a long time until the solution is at equilibrium. Describe what is going on with the sugar molecules at equilibrium.

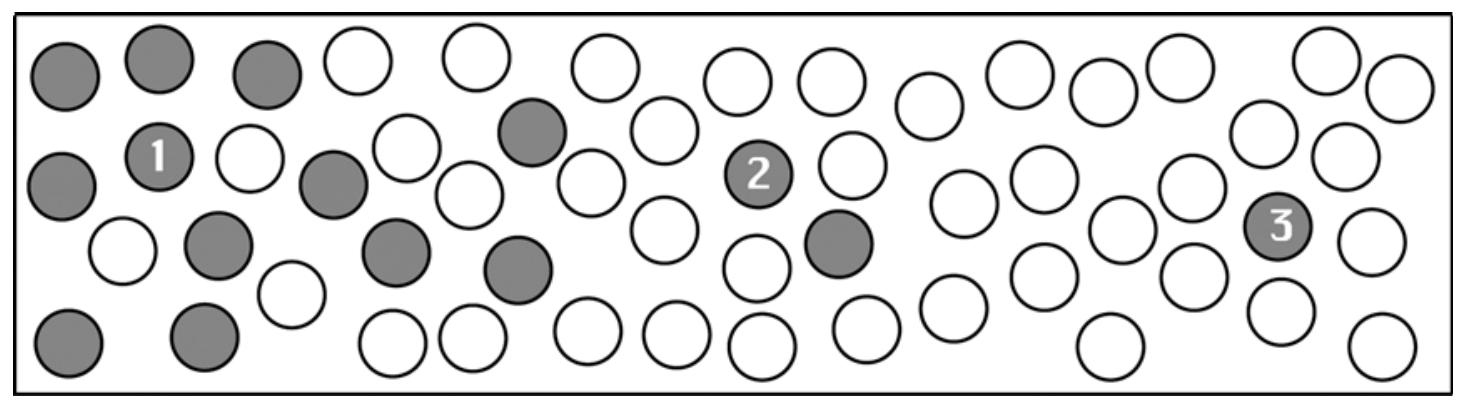

3. Imagine this is a picture of molecules frozen in time. The gray circles are uncharged molecules that are diffusing in the clear water molecules inside a tube. In the next moment (at the instant when we let the molecules move again) draw arrow(s) showing the direction(s) that each of the numbered balls would likely move. If it is likely to move in only one direction, draw a single arrow. If it might move in several different directions, draw multiple arrows and indicate how likely each direction of movement is by the size of the arrows (draw larger arrows in the more likely directions).

4. You have a shallow rectangular tray filled with water and an eyedropper with food coloring. You put one large drop of food coloring into the water on the left edge of the tray. In one or two sentences, describe:

4a. What happens to the color of the water in the tray over time?

4b. The middle of the tray starts showing some color after $1 \mathrm{~min}$. Assuming nothing else changes, how much longer will it take for the right side of the tray to start showing some color?
a. Less than 1 additional min
b. About 1 additional min
c. More than 1 additional min
d. Depends on the temperature

4c. What is the reason for the answer you gave above?

Questions 5 and 6 on our test are questions 2 and 3 on the assessment instrument written by Odom (1995).

\section{Osmosis Survey}

Mark each of the following questions True or False. Then, if false, correct the statement so it is true.

1a. Osmosis requires an impermeable membrane. T/ F

Corrected: 
1b. During osmosis, individual water molecules "sense" areas that have lesser concentrations of water and move toward them. T / F

Corrected:

1c. Once concentrations of solvent inside and outside of the cell are equal, water molecules stop flowing across the membrane. T / F

Corrected:

1d. During osmosis, the system will reach a state of equilibrium when there are approximately an equal number of solvent (water) molecules on both sides of the membrane. T / F

Corrected:

1e. A solution that is hypertonic with respect to a cell will cause the cell to grow over time. T / F

Corrected:

2. You have a cell with the following composition

3 percent salt

1 percent protein

96 percent water

3. You want to make a solution that is isotonic to the cell using only salt and water (no protein). What percent of salt would you put into the solution?

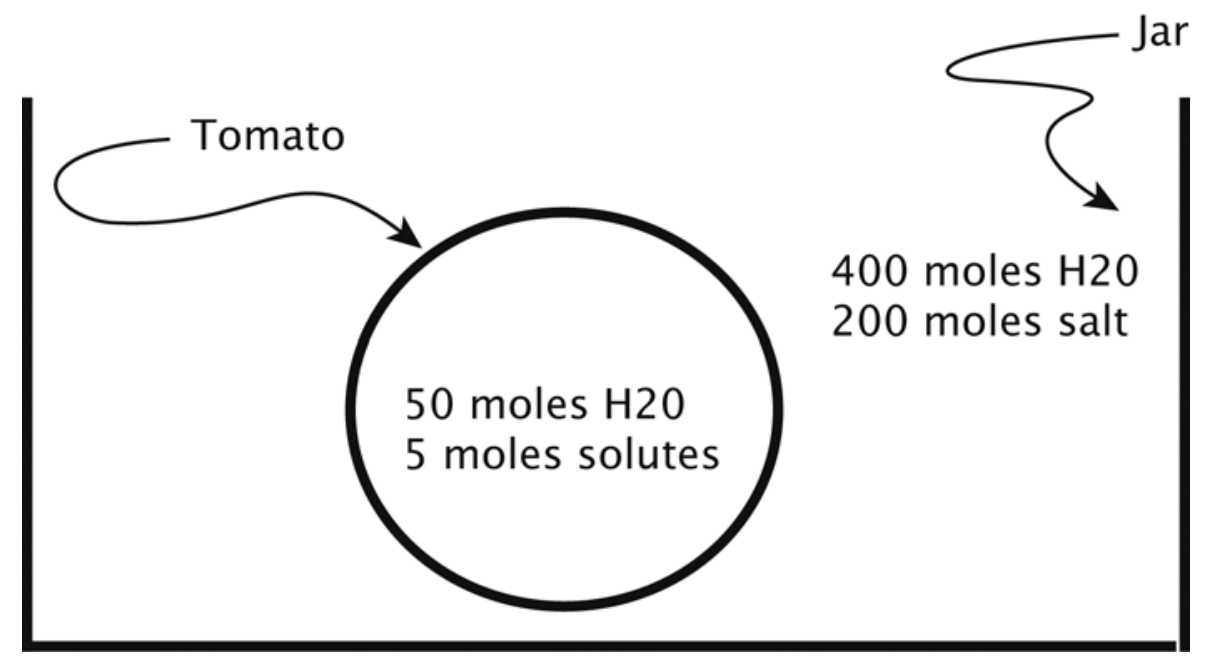

The above picture shows a tomato being pickled in a jar of brine (salt water). Assume the tomato skin lets in water, but not salt.

4a. Will the tomato increase in size, decrease in size, or stay the same size over time?

$\bigcirc$ Increase $\bigcirc$ Decrease $\bigcirc$ Same size

4b. Explain your answer.

4c. Assuming that the tomato can freely change size as molecules enter and leave, and the brine is changed periodically so the concentration of salt stays constant, how many moles of water will the tomato have by the time it is done pickling?

400 moles of water, as in the outside solution

- 40 moles of water, the ratio of solutes on outside to solutes on inside

$\bigcirc 10$ moles of water, the ratio of water to salt in the outside solution

8 moles of water, the original ratio of water on outside to water on inside

$\bigcirc$ Cannot be determined from this information

4d. Explain your answer. 
The container below has a semi-permeable membrane in the middle and a water-tight plunger on each side that can move up and down. The liquid inside has two types of molecules, shown by open and closed circles. The membrane is only permeable to the open molecules (with numbers), but is NOT permeable to the filled molecules (with letters).

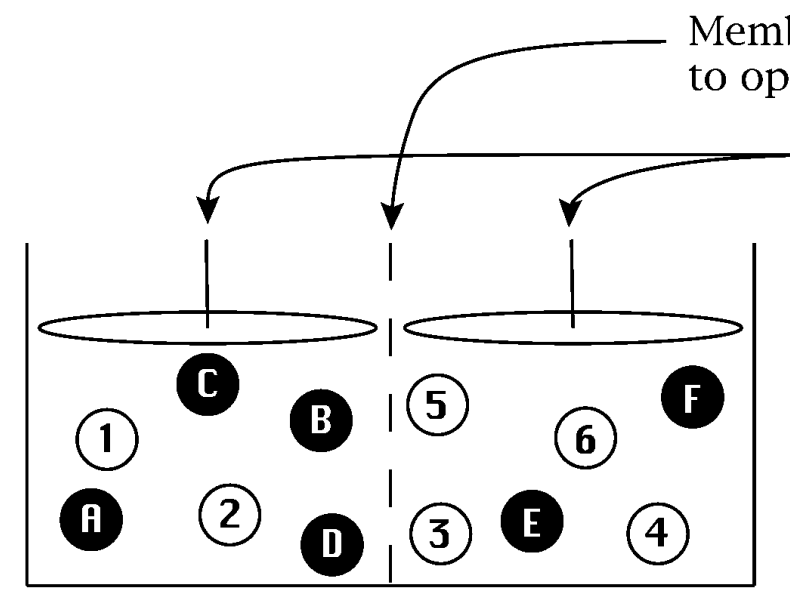

In the diagram below, draw the system as it might look when it reaches an equilibrium (no more net changes). Assume each molecule drawn represents millions of actual molecules. Number the balls in your drawing and don't forget the plungers.

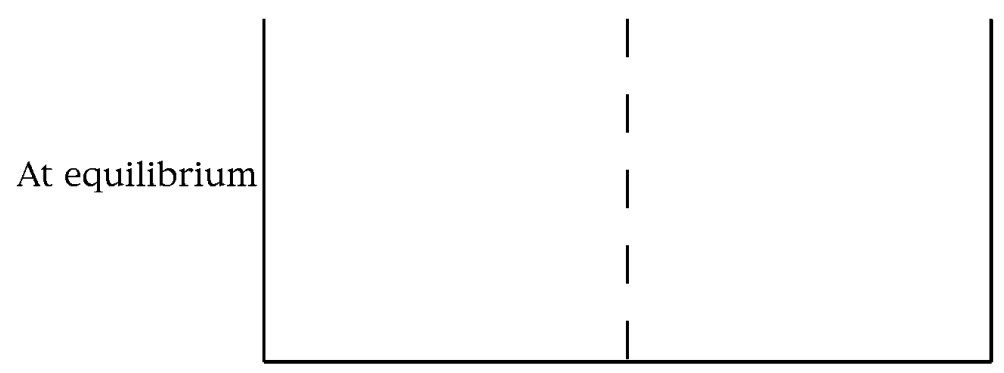

In the diagram below, draw the system as it might look a while after it reaches equilibrium. Then explain both drawing in the space on the right.

Explain your top drawings here Plungers move up and down in response to pressure

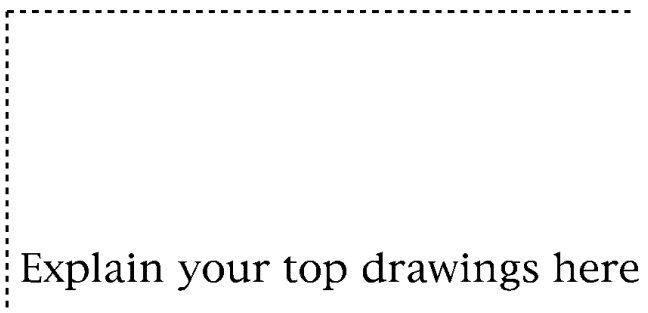

Explain your bottom drawing here
A while after equilibrium

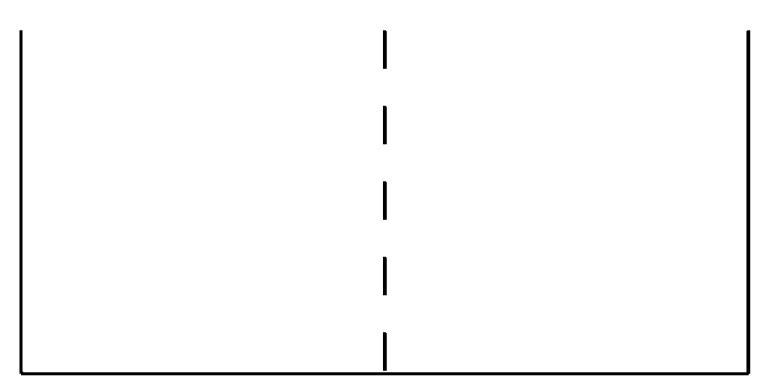

